\title{
The Modelica library AlternativeVehicles for vehicle system simulation
}

\author{
Thomas Braig ${ }^{1}$ thomas.braig@dlr.de, Holger Dittus ${ }^{1}$ holger.dittus@dlr.de, \\ Jörg Ungethüm ${ }^{2}$ joerg.ungethuem@dlr.de, Tobias Engelhardt ${ }^{1}$ tobias.engelhardt@d,dr.de \\ Deutsches Zentrum für Luft- und Raumfahrt e. V. (DLR) (German Aerospace Centre) \\ ${ }^{1}$ Institut für Fahrzeugkonzepte (Institute of Vehicle Concepts) \\ ${ }^{2}$ Institut für Technische Thermodynamik (Institute of Technical Thermodynamics) \\ Pfaffenwaldring 38-40, 70569 Stuttgart
}

\begin{abstract}
The AlternativeVehicles Library (AV) allows to calculate the energy demand and optimize the energy management for conventional and alternative vehicle concepts. The components provided focus on the simulation of alternative power trains. Therefore models of energy storages and energy converters such as electric drives and fuel cells are included.

The library was developed within the European research project Eurosyslib. The current version includes contributions of the DLR Institute of Robotics and Mechatronics and is distributed by the Bausch-Gall GmbH.

Within this paper architecture and components of the AlternativeVehicles library are described. Exemplarily fuel consumptions of a conventional vehicle and a parallel hybrid vehicle are compared.
\end{abstract}

\section{Introduction}

The activities at the Institute of Vehicle Concepts contribute to the sustainable development of technological systems for future generations of road and railway vehicles. Therefore components for energy conversion and energy recuperation are developed and integrated into research vehicles [1]. Also the scenario tool Vector21 has been developed, which allows assessing the influence of car specific fuel consumption, energy prices, taxation, customer decision etc. on the future vehicle fleet and total $\mathrm{CO} 2$ emissions [2]. System level simulations of different vehicle concepts are essential for all of these activities. Due to the lack of commercially available, flexible, appropriate tools new models have been created in Modelica.

During the last years the Modelica library AlternativeVehicles (AV) has been developed with contributions of the DLR Institute of Institute of Robotics and Mechatronics and since March 2011 it is commercially available.

\section{Overview of the AlternativeVehicles Library}

Among the vehicle concepts provided in the library are architectures of a conventional ICE powered vehicle, an electric vehicle with combustion engine based range extender, a pure electric vehicle with a high-voltage battery as energy storage, a parallel hybrid vehicle, and a fuel cell hybrid electric vehicle. To enable fast simulations of entire vehicle 
systems, mainly concentrated modeling approaches are used for the component models. The components provided focus on the simulation of alternative power trains. Therefore models of energy storages (battery, double layer capacitor) and energy converters such as fuel cells, electric motors and power electronics are included. In addition to the component models the library includes various parameter sets for component models representing technical data of real life components. Some component models are available in different detail levels which enables the user to choose an appropriate modeling approach depending on the objective of the simulations. Additional vehicle concepts can be easily created by combining the available components and developing the control strategy.

The AV is modeled in Modelica. Modelica is a simulation language which is nonproprietary, object-oriented and equation based. It allows to model complex multiphysical systems containing, e.g., mechanical, electrical, electronic, hydraulic, thermal, control, electric power or process-oriented subcomponents [3]. Each model consists of interfaces and mathematical equations (algebraic or ordinary differential equations) respectively algorithms. Physical interfaces, such as heat ports, electrical pins or mechanical flanges consist of flow and potential variables. Modelica models do not only describe the mathematical behavior of the real world system but also have the same structure. This leads to intuitively creation and understanding of the models.

To ensure the compatibility to other automotive Modelica libraries, the AV is based on the freely available VehicleInterfaces library [4]. The components are connected via different interfaces: physical connectors and signal buses (e. g. Fig. 1: flange, signal bus).

\section{Vehicle Architectures: Conventional Vehicle and Parallel Hybrid}

Several vehicle architectures are provided within the AV. In Fig. 1 and Fig. 2 the top level models of a conventional vehicle and a parallel hybrid vehicle are shown. The conventional vehicle consists of the following top level components (top level model Fig. 1): driver, control module, accessories, engine (ICE), transmission, driveline, chassis and inspector. The parallel hybrid vehicle has additionally an electric motor, high voltage battery, and a clutch between the engine and the electric motor [5].

The control module of the parallel hybrid is labeled hybrid control unit (HCU). Every top level component is connected with the control module and has its own status and control sub bus, e.g. EngineStatusBus and EngineControlBus. The status buses contain signals describing the actual state of the system and the control buses contain signals to control the system such as setpoint values (Fig. 3). 


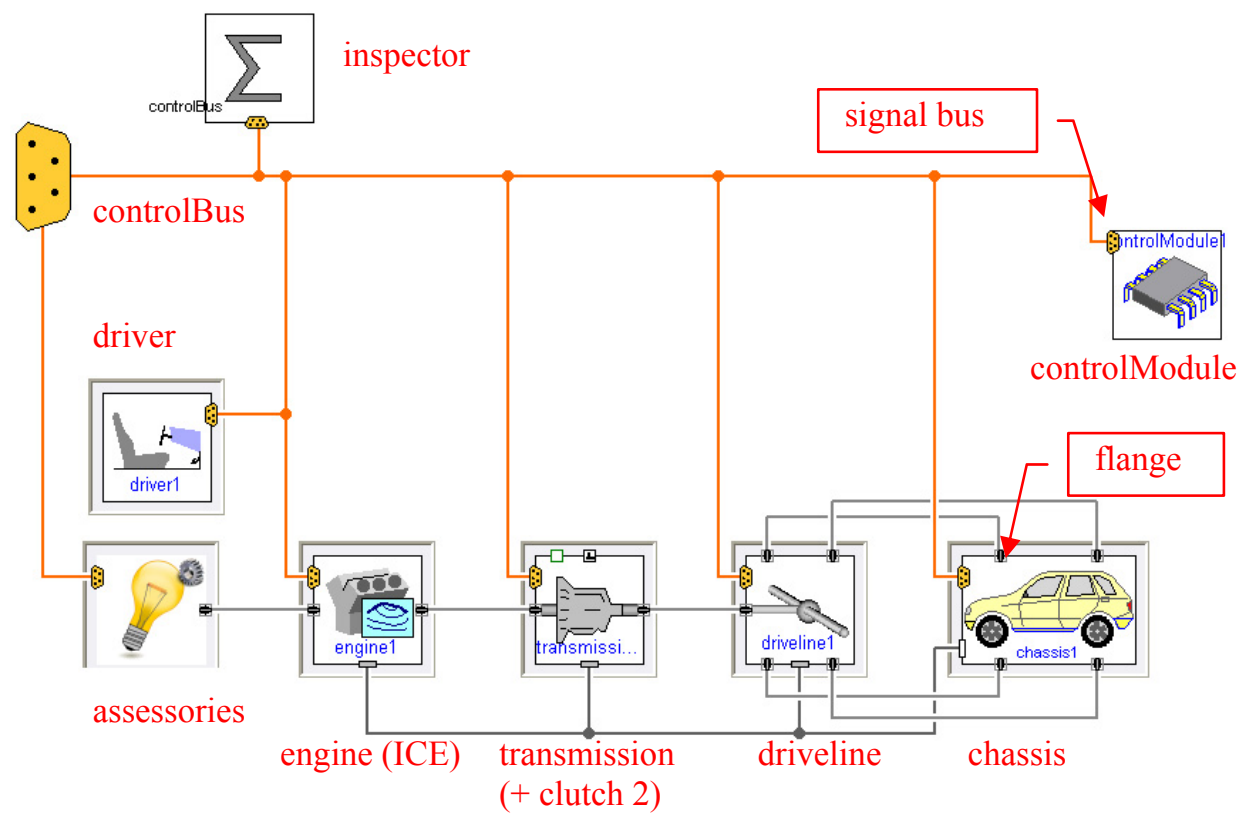

Fig. 1: Top level model of the Conventional Vehicle

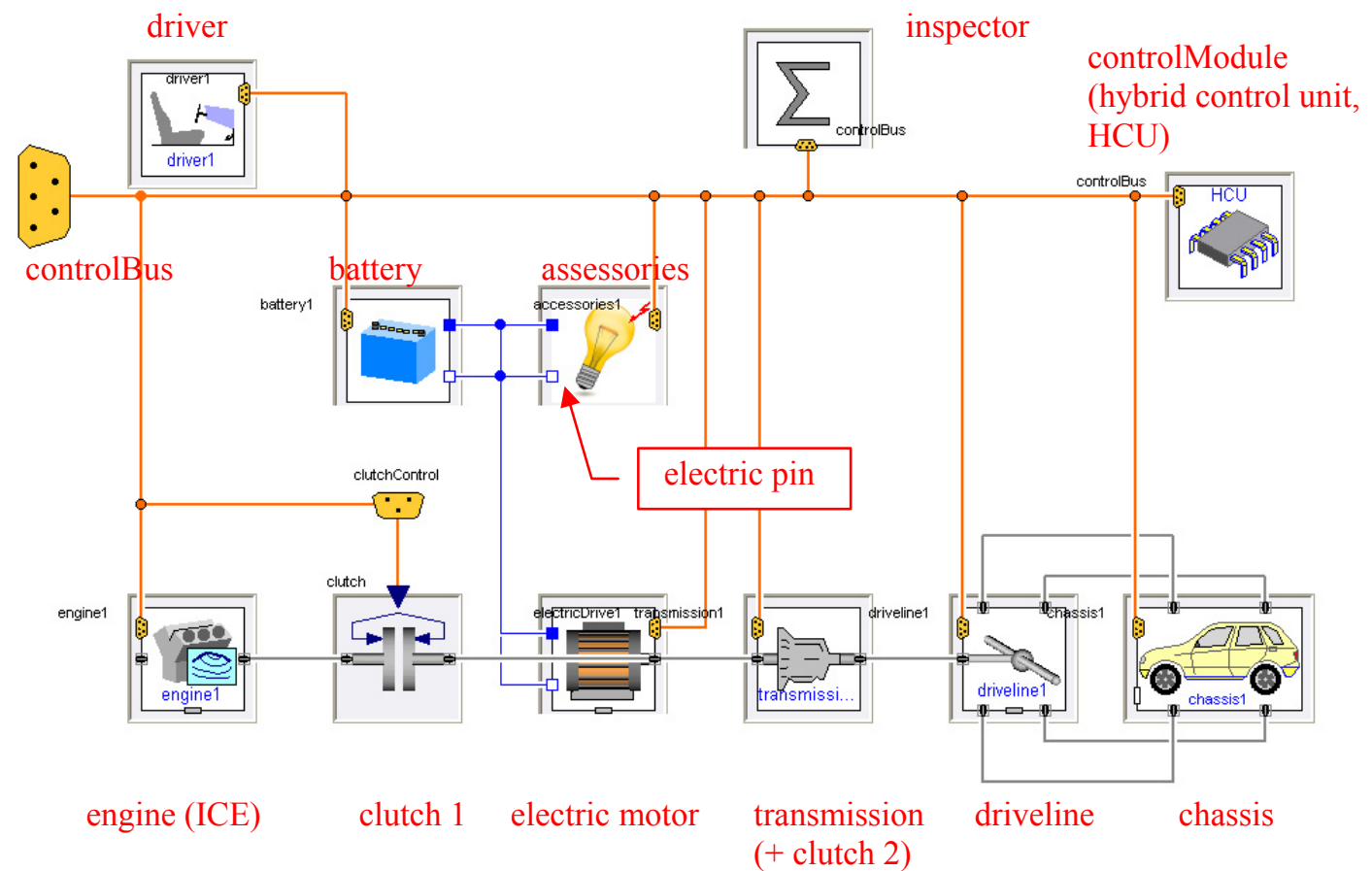

Fig. 2: Top level model of the Parallel Hybrid 
The top level components of the parallel hybrid vehicle are:

Driver: The "StandardCycleDriverManual" is a driver model with gearshift and clutch control suitable for ICE driven vehicles with manual gearshift. The core of the "StandardCycleDriverManual" model is a PI-controller which is fed by the set-point speed from the driving cycle. Clutch and gearshift control is done by several embedded submodels. The driver model includes various driving cycles (NEDC, HYZEM, FTP75, Artemis etc.).

ControlModule: The controlModule (hybrid control unit, HCU) controls the other top level components. All settings related to the operating strategy are made in the HCU. The HCU enables simulation of several kinds of hybrids with different operating strategies.

Engine: The engine is a table-based model of an internal combustion engine (ICE). Torque and fuel consumption are defined by tables, which can be adapted to the desired engine. The model optionally includes an idle speed controller, an overdrive protection and a starter motor.

Electric Motor: The "SimpleDrive4Tab" is a table-based model containing 4 tables: Torque and efficiency for both directions (motor, generator). The EM itself can be overloaded but the HCU is not designed to use this option.

Clutch 1: The clutch 1, which is controlled by the HCU, can optionally be disabled (e. g. in case of a micro hybrid)

Transmission: The "SimpleManualGear" is a model of a manual transmission. It is a combination of clutch and gearbox

Driveline: The driveline model is of a rear-wheel drive 4-wheeled vehicle. The rear differential is modeled using an ideal gear and planetary gear.

Chassis: The "TwoWheelPolynomial" is a single tracked (two wheels) model with polynomial driving resistance.

Battery: Impedance based battery model using 2-dim parameter table lookup.

Accessories: Electric driven auxiliaries represent any electric load of a vehicle. In conventional vehicles are servo motors, fans, etc. the major electric loads. In full electric vehicles also the AC system is electric. This model provides just a constant electric load.

Inspector: The inspector is used for post-processing. It calculates characteristic numbers as efficiencies or fuel consumption.

\section{Hybrid Control Unit}

The task of the hybrid control unit (HCU) is controlling the complex hybrid drivetrain. It contains the operating modes:

- Electric Driving: This mode is used in vehicles where the electric drive torque is sufficient to power the vehicle exclusively. The ICE is turned off and the vehicle is moved by the EM like an EV.

- Start-Stop: The ICE is shut down whenever the vehicle stops and is turned on again when the vehicle starts. Since the EM is more powerful than a normal starter, the ICE starts more quickly and with less noise than in conventional vehicles.

- Load Point Shifting (LPS): ICEs and especially gasoline ICEs have poor efficiency at low loads. In low load situations the EM generates electricity, charges the energy storage (e.g. battery) and rises the demanded load of the ICE. 
- Recuperation: Recuperation or regenerative braking means the recovery of kinetic energy by the EM working as a generator to charge the battery.

- Electric Boost: The EM assists the ICE to accelerate the vehicle. The EM has great torque at low angular velocity where the ICE has a weak spot. The combination of both results in a higher and more constant torque distribution. The electric boost can either occur to start the vehicle (low acceleration, startup assistance) or when high acceleration is required.

The driver is no longer in direct control of the way the vehicle delivers propulsion. In contrast to a conventional vehicle, where the gas pedal signal of the driver is passed through unchanged to the ICE, the gas pedal signal in a HEV only indicates the desired amount of propulsion. The HCU calculates how to deliver the required propulsion most efficiently. In order to do this, it uses an implemented algorithm which depends on many variables and parameters (operating strategy). The HEV is able to perform various operating modes. All operating modes depend on various conditions. The operating strategy is crucial for the efficiency and thus the fuel consumption of the vehicle. The HCU needs to calculate the most efficient operating mode at any time always considering the state of all components (e.g. maximal available engine torque, SOC of the energy storage).

The HCU consist of subunits, signal processing, sub buses of the top level components and sub buses of the subunits (Fig. 3). Some of the above mentioned operating modes are implemented in a special subunit (e.g. Start_Stop).

The Start_Stop (controller) sub unit is responsible for stopping and starting the ICE. It has three output signals: A start and a stop signal for the ICE and, since there is no starter included in the ICE and a controlLever signal for the EM. The ICE starts if it gets an engineStart signal and it is simultaneously accelerated by the EM. For shutting down, the engineStop signal is sufficient.

Starting and stopping the ICE occurs in a certain order. In the model this order is maintained using the state graph library of the modelica standard library (Fig. 4, right). 


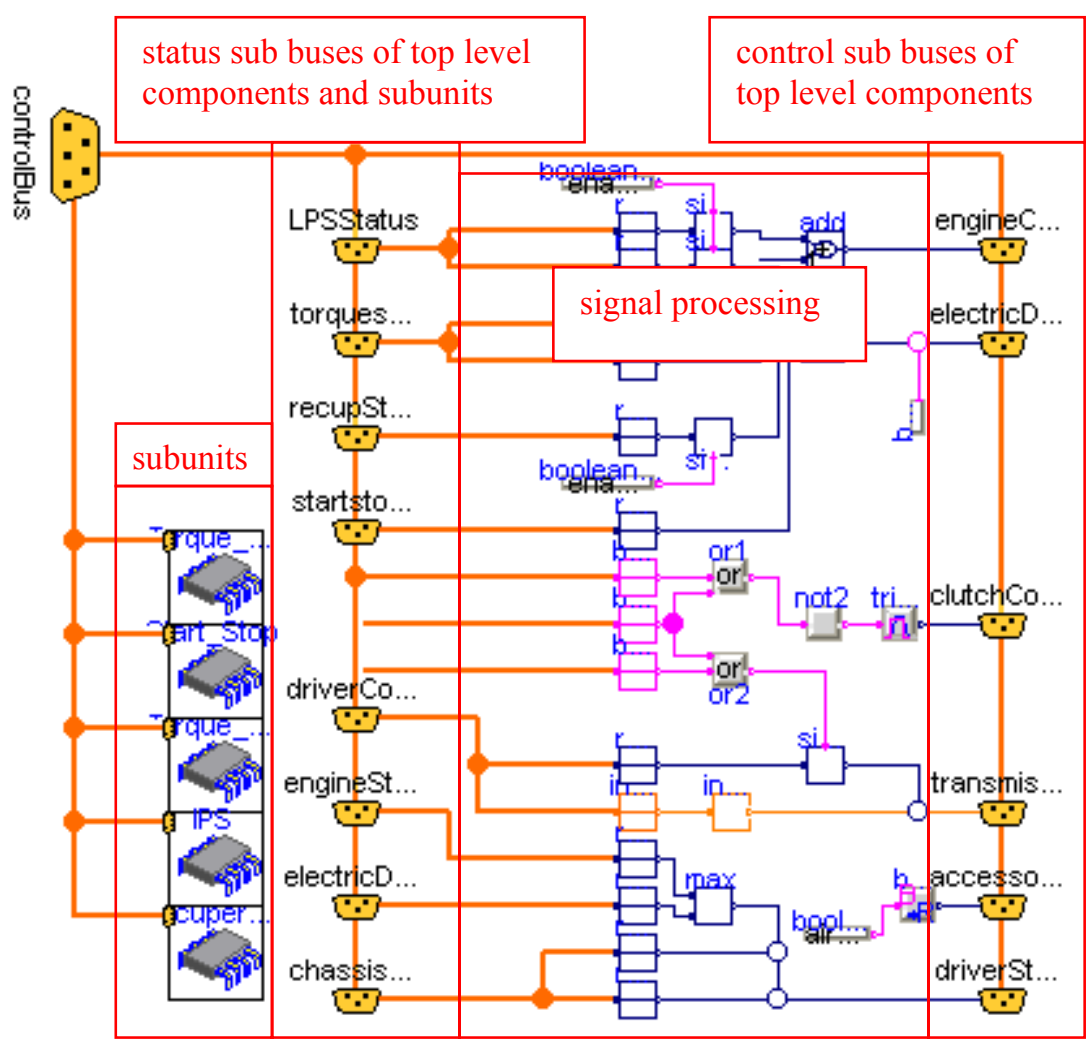

Fig. 3 Hybrid Control Unit (HCU)
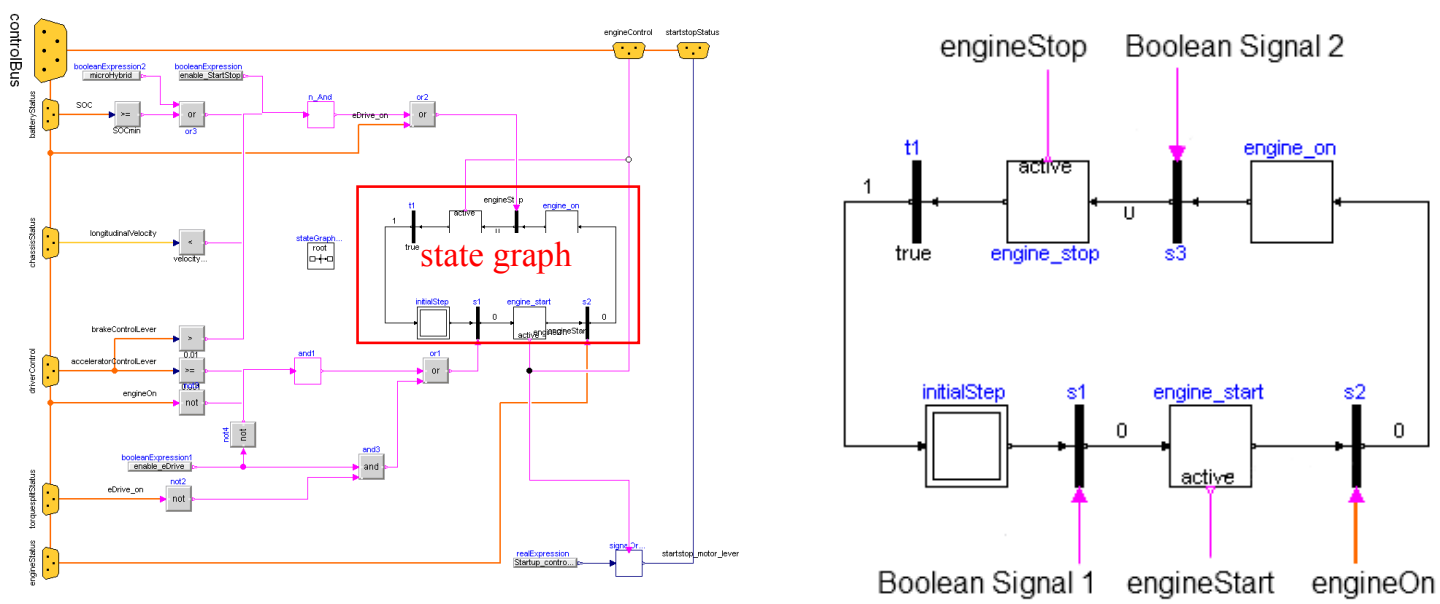

Fig. 4 left: Start_Stop sub unit, right: assignment of engineStart and engineStop signals with state graphs 


\section{$5 \quad$ Parametrized Models and Simulation Results}

In the AV parameterized models are available, e. g. a VW Golf V conventional vehicle, a DLR fuel cell hybrid electric vehicle and a DLR battery electric vehicle. The MercedesBenz S 400 BlueHYBRID (S $400 \mathrm{H}$ ) is a parallel hybrid without clutch between ICE and EM (Fig. 2, clutch 1 closed). In this car the operation modes Start-Stop, regenerative braking, and electric boost are availabel. The S $400 \mathrm{H}$ is based on the Mercedes-Benz S 350. Both vehicles are parameterized and simulated for comparison [5].

Both vehicles are driven by the M $272 \mathrm{KE}$ engine which is a naturally aspirated V6 gasoline ICE with intake-manifold fuel injection. They also share the NAG2 automatic 7speed gearbox. The EM is located between ICE and gearbox. It delivers $15 \mathrm{~kW}$ as motor and $19 \mathrm{~kW}$ as generator. The Lithium-Ion battery of the vehicle contains $0.8 \mathrm{kWh}$ and supplies the power electronics and also the electric AC compressor. A DC-DC converter is located between the $126 \mathrm{~V}$ high-voltage battery and the $12 \mathrm{~V}$ battery. The vehicle has rear wheel drive. All parameterization data are taken from literature [7][8].

Fig. 5 shows the longitudinalVelocity of the NEDC (top), the SOC of the battery (middle) and the EM-controlLever (bottom). The end value of the SOC equals the start value. The EM-controlLever value becomes positive when the vehicle is accelerating (electric boost, startup assistance) and negative when the vehicle is braking (recuperation). A positive EM-controlLever value causes a decrease of the SOC and a negative EM-controlLever value causes an increase of the SOC. If the EM-controlLever equals zero, the SOC is slightly decreasing due to the accessories $(120 \mathrm{~W})$.

For the $\mathrm{S} 350$ the result is $10.01 / 100 \mathrm{~km}$ which is very close to the fuel consumption specification of the $\mathrm{S} 350(10.1-10.31 / 100 \mathrm{~km}$ [8]). The average value of the $\mathrm{S} 400 \mathrm{H}$ is $8.561 / 100 \mathrm{~km}$. This result differs from the fuel consumption specification of the $\mathrm{S} 400 \mathrm{H}$ $(7.9-8.11 / 100 \mathrm{~km} \mathrm{[8])}$ by $5 \%$. Several reasons might lead to this overestimation, e. $\mathrm{g}$. deviations in the driving resistances or the accessories are overestimated. 

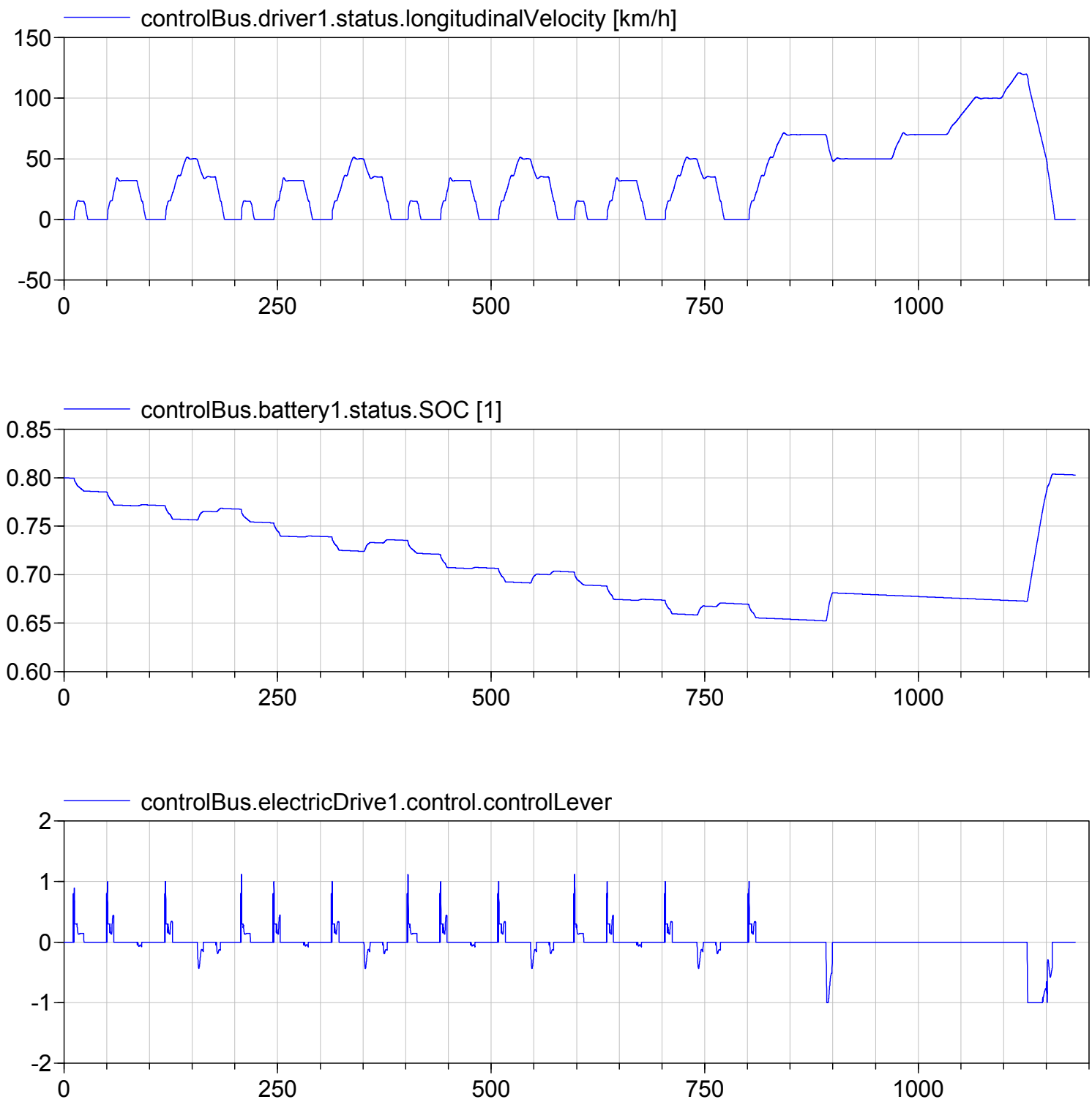

Fig. 5 Simulation results for the Parallel Hybrid S $400 \mathrm{H}$ in the NEDC: top: longitudinalVelocity, middle: SOC of the battery, bottom: EM-controlLever

\section{Conclusion}

The AV is a commercially available, open source automotive Modelica library which allows the user to model various vehicle concepts, develop operating strategies and calculate the energy demand for different driving cycles. The main contents of the AV are models and parameterized examples for several alternative vehicle architectures, energy storages and energy converters. 
In the paper the models and simulation results of a conventional vehicle and a parallel hybrid vehicle have been compared. The calculated fuel consumptions fit the values of the specifications sheet quite well. The deviations can be caused by several reasons, e. g. deviations in the driving resistance or overestimated accessories.

\section{Abbreviations}

AV AlternativeVehicles Library

DLR Deutsches Zentrum für Luft- und Raumfahrt e. V. (German Aerospace Center)

EM Electric Motor

EV Electric Vehicle

HCU Hybrid Control Unit

ICE Internal Combustion Engine

LPS Load Point Shifting

NEDC New European Driving Cycle

$\mathrm{SU} \quad$ sub unit (of the $\mathrm{HCU}$ )

\section{Literature}

[1] Braig, T.; Ungethüm, J: System-Level Modeling of an ICE-powered Vehicle with Thermoelectric Waste-Heat-Utilization, Modelica Conference, Como/Italy, 2009

[2] Mock, P.; Hülsebusch, D.; Ungethüm, J.; Schmid, S.A.: Electric vehicles - A model based assessment of future market prospects and environmental impacts. EVS24, Stavanger, Norway, May 13-16, 2009

[3] Modelica Association: Modelica and the Modelica Association. https://www.modelica.org/

[4] Dynasim, DLR, and Modelon: VehicleInterfaces Library, Version 1.1, Tutorial and Reference, 2008

[5] Engelhardt, T.: Modeling and Simulation of a Parallel Hybrid Vehicle in Modelica, Studienarbeit (unpublished), 2011

[6] Ungethüm, J.; Hülsebusch, D.; Dittus, H.; Braig, T.: Simulation von alternativen Fahrzeugentrieben in Modelica, ASIM Conference, Ulm 2010,

[7] Hofmann, P.: Hybridfahrzeuge, Springer-Verlag, Wien, 2010

[8] Vollrath, Armstrong, Schenk, Bitsche, Lamm: S 400 BlueHYBRID - First Hybird Vehicle with Li-Ion-Technology. In: 30. Internationales Wiener Motorensymposium 2009 